\title{
Functional characterization of Trip10 in cancer cell growth and survival
}

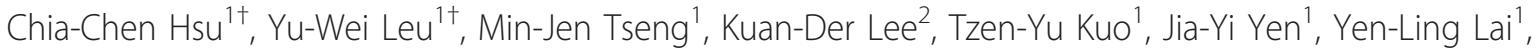 \\ Yi-Chen Hung ${ }^{1}$, Wei-Sheng Sun ${ }^{1}$, Chien-Min Chen ${ }^{3}$, Pei-Yi Chu ${ }^{4}$, Kun-Tu Yeh ${ }^{4}$, Pearlly S Yan ${ }^{5}$, Yu-Sun Chang ${ }^{6}$, \\ Tim H-M Huang ${ }^{5}$, Shu-Huei Hsiao ${ }^{1 *}$
}

\begin{abstract}
Background: The Cdc42-interacting protein-4, Trip10 (also known as CIP4), is a multi-domain adaptor protein involved in diverse cellular processes, which functions in a tissue-specific and cell lineage-specific manner. We previously found that Trip10 is highly expressed in estrogen receptor-expressing $\left(\mathrm{ER}^{+}\right)$breast cancer cells. Estrogen receptor depletion reduced Trip10 expression by progressively increasing DNA methylation. We hypothesized that Trip10 functions as a tumor suppressor and may be involved in the malignancy of ER-negative (ER-) breast cancer. To test this hypothesis and evaluate whether Trip10 is epigenetically regulated by DNA methylation in other cancers, we evaluated DNA methylation of Trip10 in liver cancer, brain tumor, ovarian cancer, and breast cancer.

Methods: We applied methylation-specific polymerase chain reaction and bisulfite sequencing to determine the DNA methylation of Trip10 in various cancer cell lines and tumor specimens. We also overexpressed Trip10 to observe its effect on colony formation and in vivo tumorigenesis.

Results: We found that Trip10 is hypermethylated in brain tumor and breast cancer, but hypomethylated in liver cancer. Overexpressed Trip10 was associated with endogenous Cdc42 and huntingtin in IMR-32 brain tumor cells and CP70 ovarian cancer cells. However, overexpression of Trip10 promoted colony formation in IMR-32 cells and tumorigenesis in mice inoculated with IMR-32 cells, whereas overexpressed Trip10 substantially suppressed colony formation in CP70 cells and tumorigenesis in mice inoculated with CP70 cells.
\end{abstract}

Conclusions: Trip10 regulates cancer cell growth and death in a cancer type-specific manner. Differential DNA methylation of Trip10 can either promote cell survival or cell death in a cell type-dependent manner.

\section{Background}

Trip10 is a scaffold protein with F-BAR, ERM, and SH3 domains. Because these domains interact with diverse signaling partners, Trip10 is involved in various cellular processes including insulin-stimulated glucose uptake, endocytosis, cytoskeleton arrangement, membrane invagination, proliferation, survival, and migration, in a tissuespecific and cell lineage-specific manner. In adipocytes, Trip10 increases glucose uptake by interacting with TC10 to regulate insulin-stimulated glucose transporter 4 (Glut4) translocation to the plasma membrane $[1,2]$.

\footnotetext{
* Correspondence: bioshh@ccu.edu.tw

+ Contributed equally

'Human Epigenomics Center, Department of Life Science, Institute of Molecular Biology and Institute of Biomedical Science, National Chung Cheng University, Chia-Yi, Taiwan

Full list of author information is available at the end of the article
}

However, in muscle cells, Trip10 inhibits glucose uptake by increasing Glut4 endocytosis [3,4]. In natural killer cells, Trip10 regulates actin cytoskeleton dynamics by interacting with WASP protein $[5,6]$, and regulates cytotoxicity by facilitating localization of microtubule organizing centers to immunological synapses [7]. Trip10 is also a regulator or modulator of cell survival after DNA damage [8] and in the human brain affected by Huntington's disease [9]. Trip10 expression is decreased in hepatocyte growth factor/scatter factor (HGF/SF)-mediated cell protection against DNA damage, but is significantly increased during hyperbaric oxygen-induced neuroprotection [10]. On the other hand, overexpression of Trip10 was observed in human Huntington's disease brain striatum, and neuronal Trip10 immunoreactivity increased with neuropathological severity in the neostriatum of

\section{Biomed Central}


Huntington's disease patients [9]. In addition, rat striatal neurons transfected with Trip10 exhibited increased cell death [9], suggesting that Trip10 is toxic to striatal neurons. These data demonstrate that the function of Trip10 in cell survival and growth is cell lineage-specific. These diverse and sometime opposing roles of Trip10 may be due in part to splicing variants, but equally important, they could be the result of Trip10 interaction with distinct signaling partners in different cell types.

Trip10 also appears to be involved in tumorigenesis and cancer progression. Enforced expression of Trip10 increases DNA damage-induced cell death in MDAMB-453 human melanoma cells and DU-145 human prostate cancer cells [8]. However, Trip10 overexpression enhances pancreatic cancer cell migration by downregulating the antitumor function of ArgBP2, suggesting that Trip10 contributes to the malignancy of pancreatic cancer [11]. In epidermoid carcinoma cells, siRNA-mediated silencing of Trip10 strongly increases epidermal growth factor receptor levels, sustains extracellular signal-regulated kinase activation, and promotes cell cycle progression into $S$ phase [12], which may contribute to excessive proliferation and tumorigenesis. In Epstein-Barr virus-transformed lymphoblastoid cell lines, blocking the NF- $\kappa \mathrm{B}$ pathway induces apoptosis and suppresses Trip10 [13], suggesting that Trip10 activation is crucial for the proliferation and survival of lymphoblasts.

DNA methylation is an epigenetic mechanism that regulates gene expression in response to intrinsic and environmental signals under normal physiological conditions (e.g., development) and pathologic conditions (e.g., cancer) [14-17]. A cohort of methyl CpG-binding proteins is recruited specifically to methylated CpG sites, where they repress transcription by limiting the access of transcription factors to the promoter. DNA hypermethylation silences tumor suppressor genes in many cancers, and the spreading of DNA hypermethylation correlates positively with tumor progression. We previously reported that Trip10 is an estrogen receptor $(E R \alpha)$ downstream target and subject to hormoneregulated epigenetic regulation [18]. In MCF7 cells, an estrogen receptor-positive $\left(\mathrm{ER}^{+}\right)$breast cancer cell line, Trip10 is strongly expressed. Loss of estrogen receptor signaling gradually reduces Trip10 expression by triggering DNA methylation. Consistently, the Trip10 promoter is hypermethylated in $\mathrm{ER}^{-}$human breast tumors, but not in $\mathrm{ER}^{+}$breast tumors.

To evaluate whether Trip10 function is regulated in a lineage-dependent manner, we used methylation-specific polymerase chain reaction (MSP) and bisulfite sequencing to assess DNA methylation of Trip10 in human primary tumor specimens and cell lines. We then overexpressed human Trip10 to evaluate its effect on colony formation and in vivo tumorigenesis in immunodeficient mice. We found that Trip10 is differentially methylated in different cancers. Overexpression of Trip10 increases colony formation and tumorigenesis of IMR-32 cells, but decreases colony formation and tumorigenesis of CP70 cells. Taken together, our results show that Trip10 expression in brain tumors, breast cancer, liver cancer, and ovarian cancer is regulated by DNA methylation, but the methylation level varies among these cancer types. Trip10 functions as a tumor suppressor or an oncogene, depending on the cell type in which it is expressed.

\section{Methods}

\section{Cell culture}

IMR-32 neuroblastoma and U87 glioma cells were grown in Dulbecco's modified Eagle's medium, CP70 ovarian carcinoma cells were grown in RPMI 1640, MCF7 breast adenocarcinoma and HepG2 liver carcinoma cells were grown in Minimum Essential Medium (MEM), and MDA-MB-231 breast adenocarcinoma cells were grown in Leibovitz's L-15. All cell cultures were supplemented with $10 \%$ fetal bovine serum, $2 \mathrm{mM}$ L-glutamine, and $100 \mu \mathrm{g} / \mathrm{ml}$ penicillin/streptomycin. Human bone marrow-derived mesenchymal stem cell (MSC) isolation and culture were performed as described previously [19]. Expansion medium consisted of MEM- $\alpha$ and $20 \%$ newborn calf serum supplemented with $100 \mu \mathrm{g} / \mathrm{ml}$ penicillin/streptomycin and $2 \mathrm{mM}$ L-glutamine. Cells were allowed to adhere overnight at $37^{\circ} \mathrm{C}$ in $95 \% \mathrm{O}_{2} / 5 \% \mathrm{CO}_{2}$. Thereafter, the culture medium was changed twice weekly. Cells were passaged at $90 \%$ confluence. All reagents were purchased from Invitrogen.

\section{Cloning of the human Trip10 promoter}

Primer sequences for human Trip10 are listed in Additional File 1: Table S1. Total RNA from MDA-MB-231 cells was purified and reverse transcribed; the resulting cDNA was used as template for PCR amplification. Purified PCR products were ligated into a cloning vector (TOPO-TA cloning kit, Invitrogen), according to the manufacturer's protocol. Inserts were confirmed by restriction digest analysis and sequencing. Trip10 was then subcloned into the pcDNA3.1 vector for transfection ( $p c D N A-T r i p 10)$.

\section{Transfectio}

The pcDNA-Trip10 plasmid $(1 \mu \mathrm{g})$ was transfected into IMR-32 and CP70 cells using DMRIE-C transfection reagent (Invitrogen), according to the manufacturer's instructions. Empty vectors were transfected into control cells as vehicle control. The antibiotic G418 $(500 \mu \mathrm{g} / \mathrm{ml})$ was added to culture medium for stable clone selection. 


\section{Bisulfite sequencing}

Genomic DNA $(0.5 \mu \mathrm{g})$ was treated with bisulfite (Zymo), PCR-amplified, cloned, and sequenced as described by Yan et al [20]. PCR primers are listed in Additional File 1: Table S1.

\section{Quantitative MSP}

Quantitative MSP (qMSP) was performed as described by Yan et al [20]. Universal methylated DNA (Millipore) served as positive control, and Col2A1 as loading control. Primers for Col2A1 were used to amplify serial dilutions $(1 / 10,1 / 100$, and $1 / 1000)$ of control bisulfiteconverted genomic DNA to generate a standard curve (Bio-Rad iQ5 real-time thermal cycler). The percentage of methylation was calculated as (florescence intensity of Trip10 amplification) $\times 100 \% /$ (florescence intensity of Col2A1 amplification). The 25- $\mu$ l qMSP reaction contain $4 \mu \mathrm{l}$ bisulfite-treated DNA template, $2 \mu \mathrm{l}$ primers (each primer mix, $2.5 \mu \mathrm{M}), 12.5 \mu \mathrm{l}$ reaction buffer $(2 \times$ SYBR Green real-time PCR Master Mix, Toyobo), and $6.5 \mu \mathrm{l}$ ddH2O. The PCR primers are listed in Additional File 1: Table S1.

\section{Immunoblotting}

Cell lysates were collected, and protein concentration was determined with a protein assay kit (Bio-Rad) using bovine serum albumin (BSA) as the standard. Proteins (40 $\mu \mathrm{g} /$ lane) was separated by gel electrophoresis and transferred to PVDF membrane. The membranes were rinsed with Tris-buffered saline Tween 20 (TBST; $20 \mathrm{mM}$ Tris, $500 \mathrm{mM} \mathrm{NaCl}, \mathrm{pH} 7.5,0.05 \%$ Tween 20) and blocked with $5 \%$ non-fat milk in TBST for $50 \mathrm{~min}$ at room temperature. After rinsing with TBST, the membrane was incubated with primary antibodies in TBST overnight at $4^{\circ} \mathrm{C}$. After rinsing with TBST, the membrane was incubated with secondary antibodies for $45 \mathrm{~min}$ at room temperature, and then rinsed again with TBST. Membranes were incubated with chemiluminescence reagent and exposed to $\mathrm{x}$-ray film.

\section{Immunoprecipitation}

To evaluate the interactions of Trip10 with endogenous Cdc42 and huntingtin in IMR-32 cells and CP70 cells, immunoprecipitation was carried out with the Catch and Release immunoprecipitation kit (Upstate) according to the manufacturer's instructions.

\section{Immunostaining}

Cells were fixed in $2 \%$ formaldehyde in phosphate buffered saline (PBS) and permeabilized in PBS containing $0.5 \%$ NP40. After blocking with horse serum (1:100 in $\mathrm{PBS})$, the cells were incubated with primary antibodies in PBS with $3 \%$ BSA. After washing with PBS, the cells were incubated with secondary antibodies in PBS with
3\% BSA. After several PBS washes, the slides were mounted with mounting medium containing 4',6diamidino-2-phenylindole (DAPI; Vector Laboratories). The primary antibodies were anti-Cdc42 (BD Transduction Laboratories), anti-huntingtin (Chemicon), and anti-Trip10 (Abcam). Fluorescein or Texas redconjugated anti-mouse or anti-rabbit IgG (Vector Laboratories) secondary antibodies were used for detection.

\section{Soft agar assay}

Soft agar was made with $0.5 \%$ bottom agar and $0.3 \%$ top agar. After plating the bottom agar, cells were mixed with top agar and plated $\left(5 \times 10^{4} /\right.$ well $)$. After 2 weeks of culture, cells were stained with $0.01 \%$ crystal violet, and the spheres (> 50 cells) in each well was counted.

\section{In vivo tumorigenesis}

Mock-transfected or Trip10-overexpressing IMR-32 and CP70 cells $\left(1 \times 10^{7}\right.$ cells $)$ were subcutaneously injected into 6-week-old nude mice (Narl:ICR-Foxn1nu).

\section{Immunohistochemistry}

Tumor masses were surgically removed from nude mice inoculated with Trip10-overexpressing IMR-32 or CP70 cells. The tumor specimens were embedded in paraffin and cut into $4-\mu \mathrm{m}$ sections or embedded in OCT and cut into $12-\mu \mathrm{m}$ sections on a cryostat (Leica). Sections were stained with hematoxylin and eosin.

\section{Chromatin immunoprecipitation (ChIP)}

ChIP assay was performed as described by Jin et al [21].

\section{Human subjects}

Human cancer tissue collection followed IRB regulations as mandated by ChangHua Christian Hospital, Taiwan. Isolation and characterization of human MSCs were conducted according to IRB regulations at Chang-Gung Memorial Hospital, Taiwan.

\section{Animal studies}

The use of mice followed the regulations and protocols reviewed and approved by the Institutional Animal Care and Use Committee at National Chung Cheng University.

\section{Results \\ Trip10 is differentially methylated in human cancer cell lines and primary tumor specimens}

We first compared DNA methylation at the Trip10 promoter and first exon in cancer cell lines and somatic stem cells (MSCs) from normal human adults by bisulfite sequencing and qMSP. The Trip10 promoter was either unmethylated or undermethylated in MSCs and CP70 ovarian cancer cells as revealed by bisulfite 
sequencing, but the same sequence was moderately methylated in breast cancer cells (MCF7 and MDA-MB231) and liver cancer cells (HepG2). Heavy methylation was seen in brain tumor cells (IMR-32 and U87) (Figure 1A left, Additional File 1: Figure S1). Methylation of the Trip10 first exon determined by MSP was similar to the pattern observed in the promoter region, in which methylation was undetectable in MSCs, slightly methylated in CP70, moderately methylated in MCF7, MDAMB-231 and HepG2 cells, but hypermethylated in IMR-32 and U87 cells (Figure 1A right). In our previous study, expression of Trip10 during MSC-to-lineagespecific differentiation is also subjected to histone medications [22], thus promoter association with histone 3 lysine 4 trimethylation (H3K4me3, active histone mark) and histone 3 lysine 27 trimethylation (H3K27me3, repressive mark) were analyzed by chromatin immunoprecipitation (ChIP). As shown in Figure 1B, all putative ER, AML- $1 \alpha$, and CREB binding sites on Trip10 promoter were enriched for H3K4me3, but not H3K27me3, confirming that Trip10 expression is regulated by both DNA methylation and histone modification.
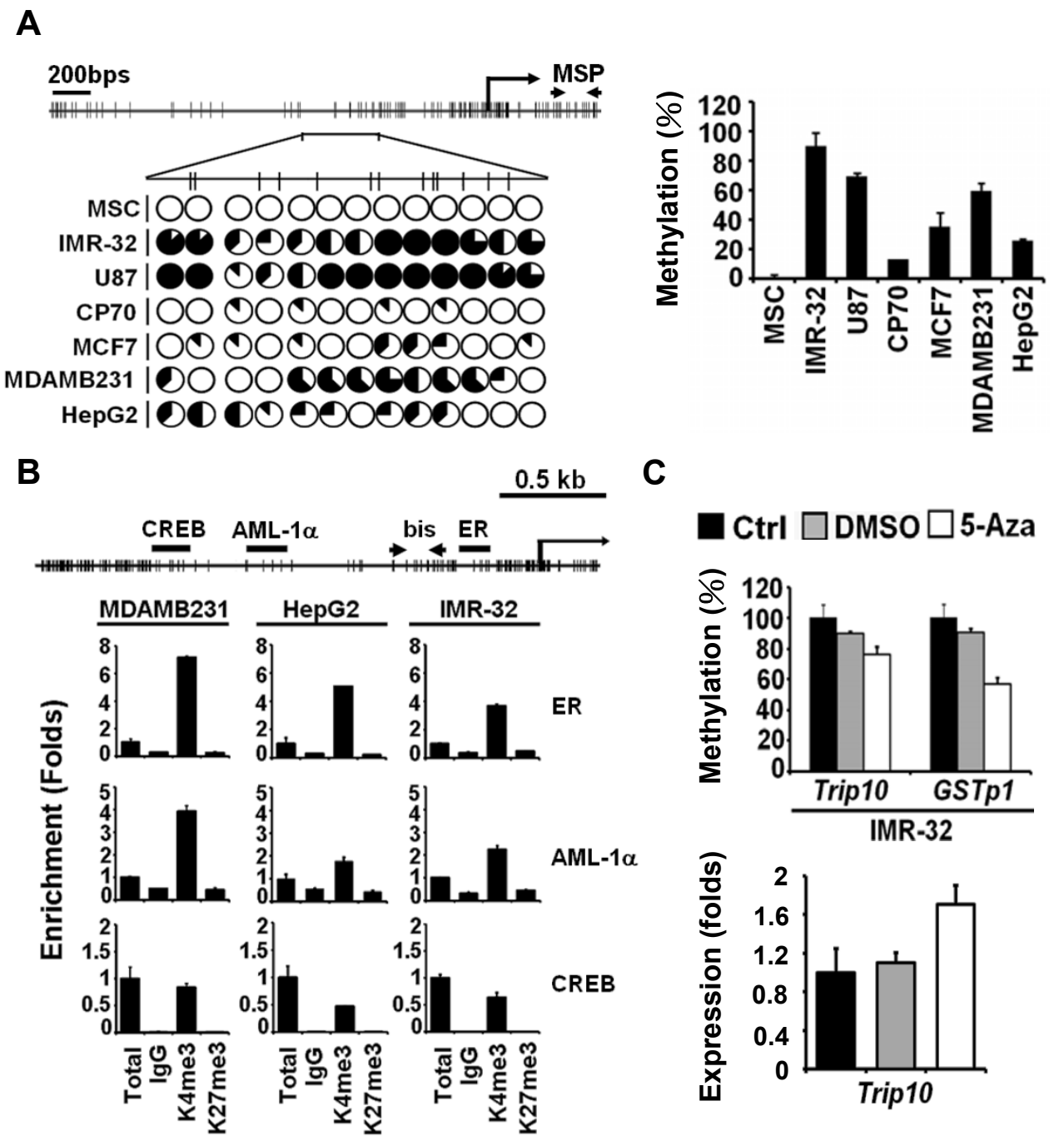

C

Ctrl $\square$ DMso $\square$ 5-Aza
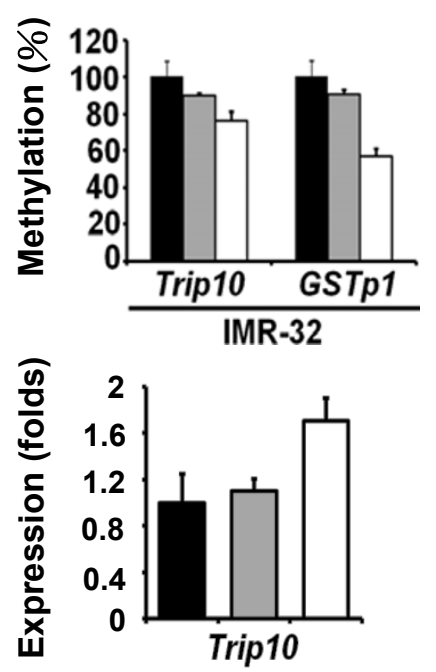

Figure 1 Epigenetic regulation of Trip10. (A) Bisulfite sequencing (left) and qMSP (right) shows TripP10 methylation in various cancer cell lines. CpG locations are indicated as vertical bars in the promoter and first exon of Trip10 (top). Arrows mark the location of MSP primers. Open circles indicate unmethylated $\mathrm{CpG}$ sites, and circles filled to varying degrees reveal the percentage of methylation at specific CpG sites. Results of eight clones from each cell line are presented. For qMSP, Col2A1 was used as loading control. (B) H3K4me3 and H3K27me3 association at Trip10 promoter were demonstrated by ChIP analysis. CREB, AML-1 $\alpha$, and ER transcription factor binding sites are shown with individual CpG sites (short vertical bars). Arrows indicate the bisulfite sequencing region shown in (A). All three transcription factor binding sites were associated with H3K4me3, but not H3K27me3. (C) DNA demethylation. IMR-32 cells treated with 5-Aza (20 $\mu$ M) or DMSO (vehicle) were analyzed by qMSP and QRT-PCR. Col2A1 served as loading control for GMSP, and GAPDH served as loading control for qRT-PCR. 
A comparison of endogenous Trip10 mRNA expression in these tested cell lines is correspondingly shown in Additional File 1: Figure S2A. To further evaluate the role of DNA methylation, IMR-32 cells were treated with 5-aza-2'-deoxycytidine (5-Aza), which appeared to suppress DNA methylation in GSTp1 and slightly decrease Trip10 DNA methylation in the first exon region (Figure $1 \mathrm{C}$ upper panel). In a good support of the MSP results, Trip10 mRNA levels were increased by 5 -Aza in IMR-32 cells as compared to controls (Figure 1C lower panel), demonstrating that the Trip10 expression is regulated epigenetically and differentially by both DNA methylation and histone modification in a cell type-specific manner.

To determine Trip10 methylation in vivo, we examined Trip10 promoter methylation in human breast cancer and liver cancer specimens and adjacent nontumor tissues. As illustrated in Figure 2 Trip10 was hypermethylated in breast cancer (Figure 2A), but hypomethylated in liver cancer (Figure 2B). Together, these data demonstrate that Trip10 is subject to epigenetic modification by DNA methylation in breast cancer and liver cancer tumorigenesis. Aberrant DNA methylation of Trip10 occurs in vivo and may contribute to neoplasm development.

Trip10 interacts with Cdc42 and huntingtin in both IMR32 and CP70 cells

Because Trip10 is differentially methylated in different types of cancer (Figure 1), we speculated that Trip10 functions in cell type-specific manner. Trip10 was thus cloned and overexpressed in IMR-32 and CP70 cells. Consistent with the qMSP results, endogenous Trip10 protein was undetectable in control IMR-32 cells by Western blot (Figure 3A, top), but weakly expressed in control CP70 cells (Figure 3B, top). Immunoprecipitation experiments showed that $\mathrm{Cdc} 42$, but not huntingtin, was expressed in IMR-32 cells (Figure 3A, center). In contrast, huntingtin was highly expressed in CP70 cells, whereas Cdc42 was expressed at low levels (Figure 3B, center). Overexpression of the Trip10 gene substantially increased cytosolic Trip10 protein and mRNA levels in both cell types (Figure 3 bottom, Additional File 1: Figure S2B). Moreover, huntingtin and Cdc42 were increased as well. Immunostaining results support the immunoprecipitation findings (Figure 3 bottom).

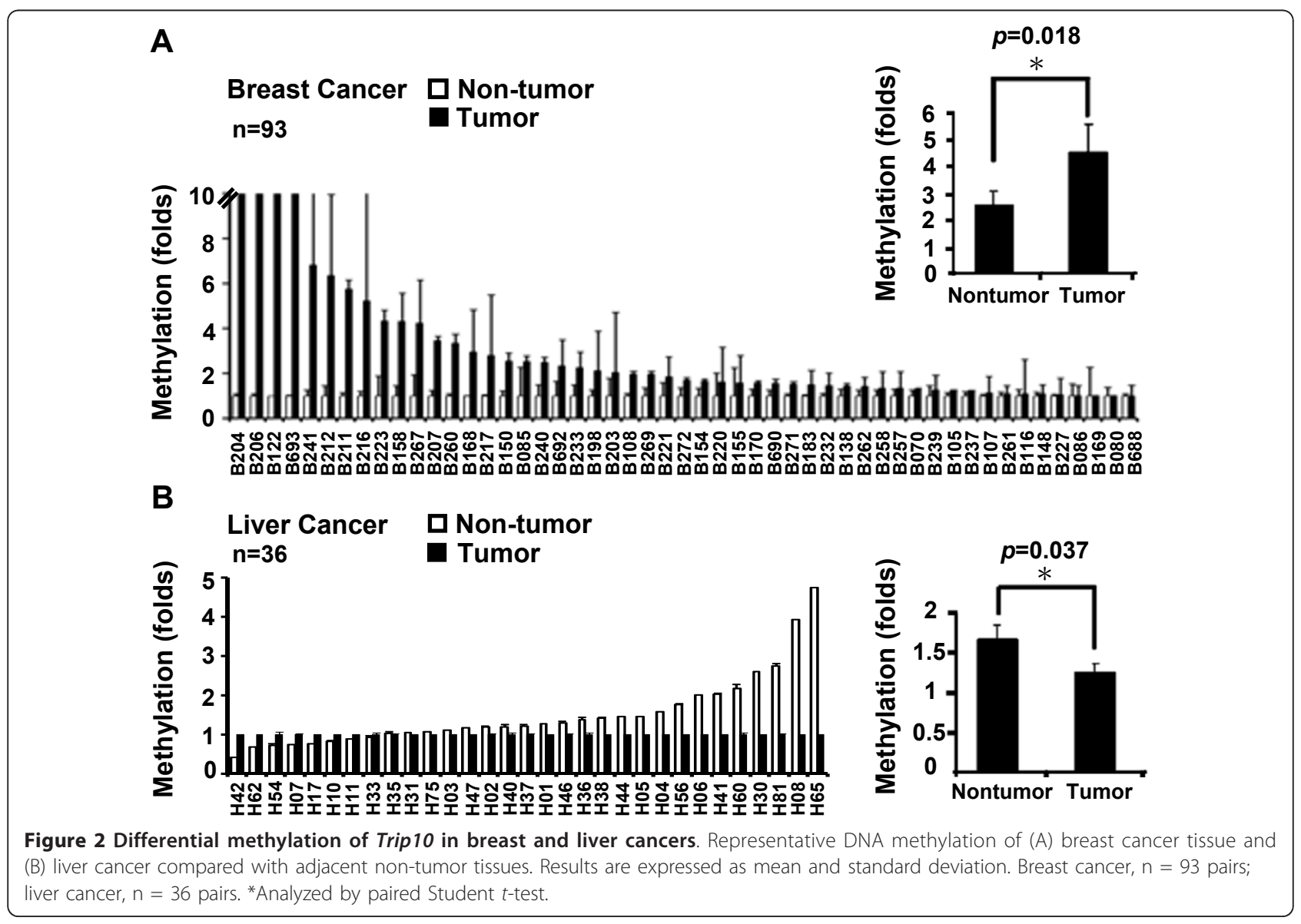




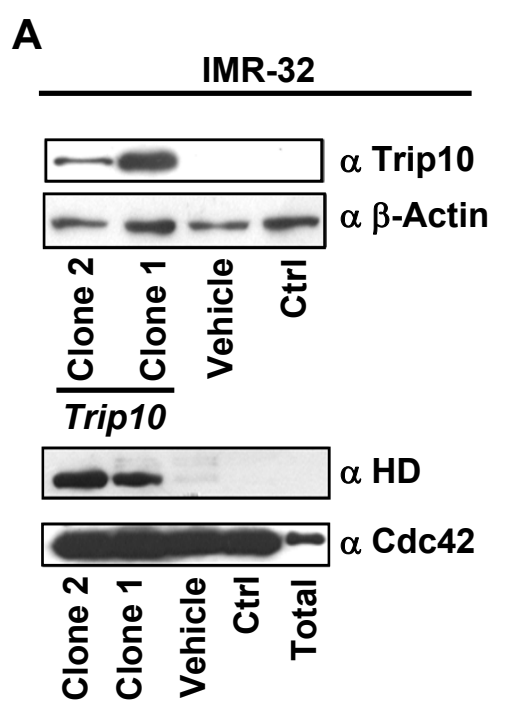

B

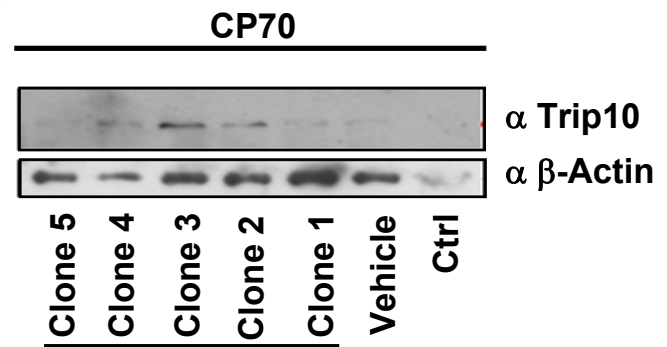

Trip10

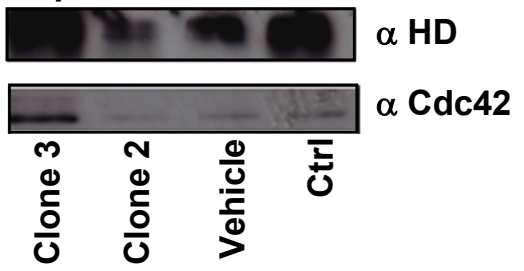

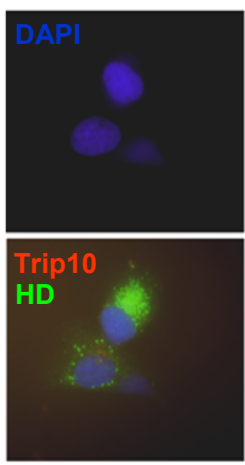

Ctrl

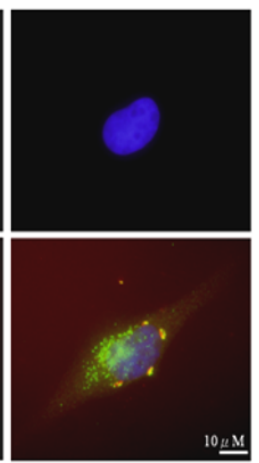

Trip10

Clone 1
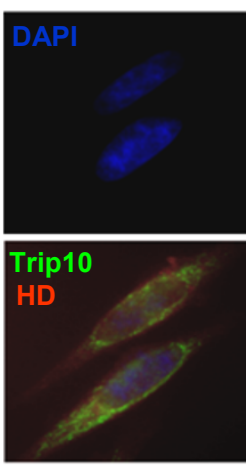

Ctrl

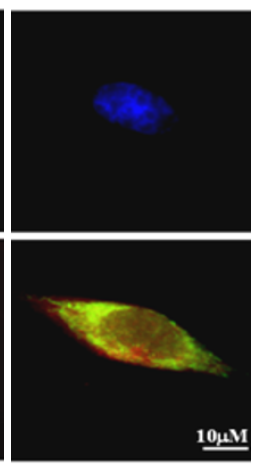

Trip10

Clone 3

Figure 3 Trip10 interacts with both Cdc42 and huntingtin (HD) and shows cell type-specific localization. Trip10 was cloned and transfected into (A) IMR-32 cells and (B) CP70 cells; individual colonies were selected and analyzed by Western blot (top panels). Interactions of Trip10 with Cdc42 and HD were analyzed by immunoprecipitation. After immunoprecipitation of Trip10, the protein complex was probed with Cdc42 and HD antibodies (middle panels). Immunostaining (bottom panels) show the distribution of Trip10 and HD. Vehicle: empty vector only; Ctrl: transfection agent only.

These results demonstrate that Trip10 associates with Cdc42 and huntingtin in IMR-32 cells and CP70 cells, but the differential expression of these proteins may lead to activation of different signalling pathways.

Trip10 promotes or suppresses in vitro colony formation and in vivo tumorigenesis in a cell type-dependent manner

Because Trip10 has been reported to regulate diverse functions and is differentially expressed in IMR-32 and CP70 cells, we next investigated the effects of overexpressed Trip10 in cell proliferation and survival. The soft agar assay was performed to evaluate in vitro colony formation. Overexpression of Trip10 promoted colony formation in IMR-32 cells (Figure 4A), but strongly inhibited colony formation in CP70 cells (Figure 4B). Both control and Trip10-overexpressing cells were then inoculated into nude mice to determine the in vivo effect of Trip10 on tumorigenesis. Consistent with results from the colony formation assay, IMR-32 cells overexpressing Trip10 formed tumors, some of which metastasized. In contrast, mice inoculated with control CP70 cells rapidly developed tumors, but tumors were not detected in mice inoculated with Trip10-overexpressing CP70 cells. These data demonstrate that Trip10 can either promote or inhibit tumorigenesis depending on the cell type in which it resides.

In Figure 3 we have demonstrated that Trip10 differentially associates with Cdc42 and huntingtin in IMR-32 cells and CP70 cells, we speculated that the differential expression of these proteins may lead to activation of different signalling pathways and contribute to the opposite oncogenic and tumor suppressive effect of Trip10. Because PI3K/Akt and MAPK pathways are 


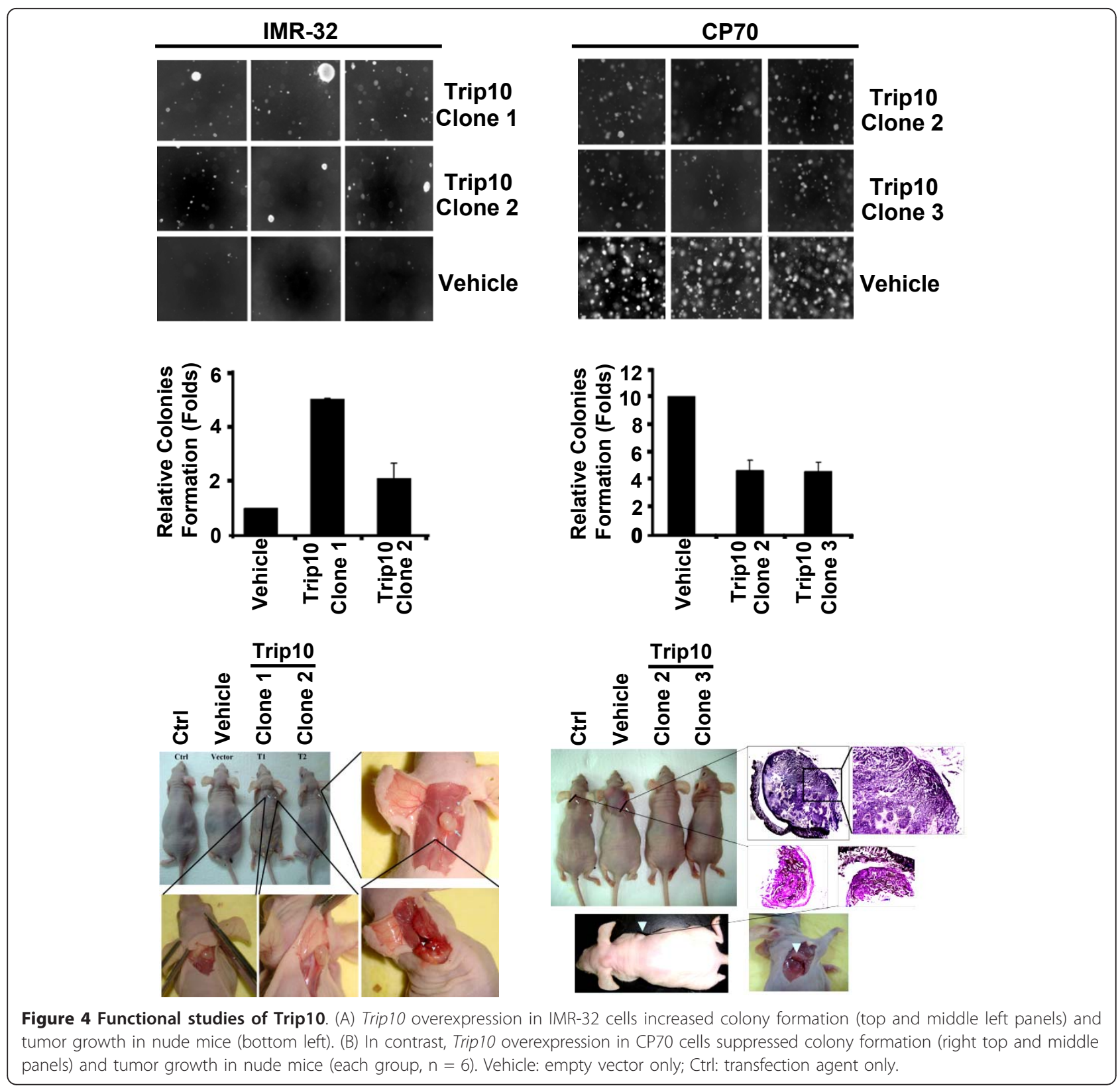

often aberrantly activated in tumor cells, and they are reported to be associated with $\mathrm{Cdc} 42$ and huntingtin [12,23-25], thus we performed qRT-PCR to determine the mRNA expression of $A k t$ and MAPK14 (encoding p38 MAPK) in Trip10-overexpressed CP70 and IMR-32 cells. Expression of $A k t 1, A k t 2$, and MAPK14 were elevated in Trip10-overexpressed cells, and the expression levels of these signalling components exhibited a positive correlation with endogenous Trip10 expression, in which more endogenous Trip10 expression is associated with greater Akt1, Akt2, and MAPK14 expression in CP70 cells as compared to the IMR-32 cells (Additional File 1: Figure S2B). Interestingly, $A k t 3$ expression is much lower in CP70 than in IMR-32 cells, furthermore, overexpression of Trip10 increased Akt 3 expression in IMR-32 cells, but not in CP70 cells. These data imply that distinct signalling components may have profound effect in the cell type-specific functions of Trip10.

\section{Discussion}

Trip10 was initially identified as a Cdc42-interacting protein involved in GLUT4-mediated glucose uptake in adipocytes and muscle cells, but Trip10 is now known to have diverse functions in wide variety of cell types. We previously identified Trip10 as an ER $\alpha$ target gene [21]. In $\mathrm{ER}^{+}$breast tumor cells, DNA methylation of 
Trip10 was not detectable; however, disrupting ER signalling caused a time-dependent increase in DNA methylation of Trip10 and reduced mRNA levels [18]. Trip10 is consistently unmethylated in $\mathrm{ER}^{+}$breast tumors but hypermethylated in ER ${ }^{-}$breast tumors. Because ER ${ }^{-}$breast cancer is generally more malignant than $\mathrm{ER}^{+}$breast cancer, these data suggest that Trip10 hypermethylation promotes tumorigenesis. In the present study, we report that Trip10 expression is epigenetically regulated by DNA methylation and histone modification in a cell type-specific manner. Among the cell lines we examined, the DNA methylation level of Trip10 (from highest to lowest) was: brain tumor cells (IMR-32 and U87) > breast tumor cells (MCF7 and MDA-MB-231) > liver cancer cells (HepG2) > ovarian cancer cells $($ CP70) $>$ MSCs (Figure 1A). Similar methylation patterns were observed in tumor specimens, Trip10 was hypermethylated in breast cancer but hypomethylated in liver cancer compared to adjacent nontumor tissues (Figure 2). Interestingly, while the Trip10 promoter was methylated in IMR-32, MDA-MB-231, and HepG2 cells, several putative transcription factor binding sites (ER, AML- $\alpha$, and CREB) were enriched for H3K4me3, association with H3K27me3 was contrarily low (Figure 1B). The expression levels of endogenous Trip10 mRNA in these cell lines (Additional File 1: Figure S2A) suggest that DNA methylation may interfere with H3K4me3 binding to the Trip10 promoter in these cells.

Functional assays reveal that Trip10 plays opposing roles in IMR-32 and CP70 cells, which may be due to differential expression of its interaction partners, thus activating different signalling pathways. The cellular localization of Trip10 also varies depending on the cell type. In COS7 and human macrophages, Trip10 is widely distributed in the cell in a "meshwork-like structure" [6]. In a skeletal muscle cell line, endogenous Trip10 is found in both the cytosol and perinuclear space, and its expression level is similar in immature myoblasts and differentiated myotubes [3]. In human brains, immunoexpression of Trip10 is detected in the nucleus and cytoplasm of neurons, activity and nuclear distribution are higher with more severe Huntington's disease [9].

In the present study, Trip10 was only sporadically in the cytosol and perinuclear region of IMR-32 control cells, but was more evenly distributed in the cytosol of CP70 control cells (Figure 3 immunostaining). Overexpression of Trip10 in IMR-32 cells caused Trip10 and huntingtin to colocalize and form perinuclear foci. In contrast, while overexpression of Trip10 in CP70 cells also increased huntingtin levels, both proteins remained in the cytosol without apparent foci formation. Western blot and immunoprecipitation studies revealed that both
IMR-32 and CP70 cells express huntingtin and Cdc42, but $\mathrm{Cdc} 42$ was more strongly expressed in IMR-32 cells (Figure 3A), whereas huntingtin was more strongly expressed in CP70 cells (Figure 3B), even when Trip10 was overexpressed. Cdc42 is involved in migration; therefore, strong Cdc42 expression in IMR-32 cells may cause them to become more invasive, possibly explaining the enhanced in vitro colony formation and in vivo tumorigenesis and metastasis in mice inoculated with Trip10-overexpressing IMR-32 cells (Figure 4A). On the other hand, huntingtin increases cell death by promoting apoptosis. Thus, high levels of huntingtin in Trip10overexpressing CP70 cells may lead to cell death, as shown by the lower rates of colony formation and tumorigenesis (Figure 4B).

Dysregulated signalling pathway is a key factor contributing to tumorigenesis and progression. In the present study, we found expression of endogenous $A k t 1, A k t 2$ and $p 38$ correlates with endogenous Trip10 expression, in which greater Trip10 expression in CP70 cells is accompanied with more $A k t 1 / 2$ and $p 38$ expression in this cell type. Overexpression of Trip10 leads to concomitantly up-regulation of $A k t 1 / 2$ and $p 38$ in both cell types, implicating that both PI3K/Akt and p38 MAPK pathways are involved in Trip10-mediated cellular behaviours. Interestingly, $A k t 3$ exhibits a distinct expression pattern. Expression of $A k t 3 \mathrm{mRNA}$ is higher in IMR-32 cells as compared to CP70 cells. Overexpression of Trip10 only promotes $A k t 3$ expression in IMR-32 cells but not in CP70, implicating that Akt3 may not be a key signalling component in CP70 cells, but may be important for tumorigenesis of IMR-32 cells. On the other hand, because amplification of $A k t 3$ has also been reported in glioblastoma [26], we reason that elevated Akt3 expression may be crucial for brain tumor formation and progression. Functional studies of the three Akt family members have revealed that they are not redundant and each fulfills unique roles [27]. Thus lack of $A k t 3$ expression along with high level of endogenous huntingtin in CP70 cells may be the determinant factors of Trip10-induced tumor suppression. In contrast, amplified Akt3 and Cdc42 may collaborate with Trip10 to trigger tumorigenesis In IMR-32 cells.

We do not rule out the possibility that specific isoforms of Trip10 are active in different cell types. In adipocytes, inactive Trip10 (CIP4/2) decreases Glut4 translocation to the plasma membrane [2], whereas in skeletal muscle cells, depletion of Trip10 (CIP4a) enhances insulin-stimulated glucose uptake by suppressing Glut4 endocytosis [3]. This difference can be explained, in part, by the fact that CIP4a does not contain the TC10-binding domain. Therefore, the differential effects of Trip10 in IMR-32 cells and CP70 cells may result from different isoforms in these two cell 
types, which recruit different interacting proteins. On the other hand, Trip10 directly interacts with WASP family verprolin-homologous protein (WAVE1) in a pancreatic cancer cell line and enhances its phosphorylation by the cytosolic tyrosine kinase c-Abl [11]. Trip10 itself is also subject to phosphorylation by c-Abl and dephosphorylation protein tyrosine phosphatase containing a PEST domain (PTP-PEST) [11]. Thus IMR-32 and CP70 cells may be equipped with different signaling pathways to regulate Trip10 activity and function.

Taken together, our data demonstrate that Trip10 expression is regulated by both DNA methylation and H3K4me3. Trip10 can enhance tumorigenesis or act as tumor suppressor depending on the cell type in which it is expressed.

\section{Conclusions}

Here we report that Trip10 is differentially methylated in different types of cancer cell lines and tumors. Analysis of histone modification in MDA-MB-231, HepG2, and IMR-32 cells demonstrated that Trip10 is associated with H3K4me3, but not H3K27me3. Trip10 can be oncogenic or tumor suppressive, increasing IMR-32 cell proliferation and inhibiting CP70 cell proliferation. The cell type-specific effect may be due, in part, to different cellular signalling partners recruited by Trip 10.

\section{Additional material}

Additional file 1: Supplementary materials. Additional file contains the supplementary materials which include: Supplementary Figures S1 to S2 and Supplementary Table S1.

\section{Abbreviations \\ Trip10: thyroid hormone receptor interactor 10; MSC: mesenchymal stem cell; 5-Aza: 5-aza-2'-deoxycytidine; H3K27me3: histone 3 lysine 27 trimethylation; H3K4me3: histone 3 lysine 4 trimethylation.}

\section{Acknowledgements}

This work was supported by NRPGM and NSC (NSC-98-3112-B-194-001, NSC97-2320-B-194-003-MY3, NSC-96-2320-B-194-004, and NSC-95-2320-B-194003) in Taiwan.

\section{Author details \\ ${ }^{1}$ Human Epigenomics Center, Department of Life Science, Institute of Molecular Biology and Institute of Biomedical Science, National Chung Cheng University, Chia-Yi, Taiwan. ${ }^{2}$ Chang Gung Memorial Hospital, Chia-Yi, Taiwan. ${ }^{3}$ Division of Neurosurgery, ChangHua Christian Hospital, ChangHua, Taiwan. ${ }^{4}$ Department of Pathology, ChangHua Christian Hospital, ChangHua, Taiwan. ${ }^{5}$ Division of Human Cancer Genetics, Department of Molecular Virology, Immunology, and Medical Genetics, and the Comprehensive Cancer Center, The Ohio State University, Columbus, OH 43210, USA. ${ }^{6}$ Graduate Institute of Basic Medical Sciences, Chang Gung University, Tao- Yuan, Taiwan.}

\section{Authors' contributions}

YWL and $\mathrm{SHH}$ designed the study and drafted the manuscript. $\mathrm{CCH}, \mathrm{YWL}$, YLL and YCH carried out the MSP and bisulfite sequencing. CCH carried out the ChIP PCR. MJT cloned the human Trip10. TYK and JYY participated in immunoprecipitation and immunostaining. $\mathrm{CCH}$ and WSS carried out colony formation assay. CMC, PYC and KTU performed the immunohistochemistry. PSY, YSC, and THH helped to draft the manuscript. All authors read and approved the final manuscript.

\section{Competing interests}

The authors declare that they have no competing interests.

Received: 26 September 2010 Accepted: 7 February 2011 Published: 7 February 2011

\section{References}

1. Lodhi IJ, Chiang SH, Chang L, Vollenweider D, Watson RT, Inoue M, Pessin JE, Saltiel AR: Gapex-5, a Rab31 guanine nucleotide exchange factor that regulates Glut4 trafficking in adipocytes. Cell Metab 2007, 5:59-72.

2. Chang L, Adams RD, Saltiel AR: The TC10-interacting protein CIP4/2 is required for insulin-stimulated Glut4 translocation in 3T3L1 adipocytes. Proc Natl Acad Sci USA 2002, 99:12835-12840.

3. Hartig SM, Ishikura S, Hicklen RS, Feng Y, Blanchard EG, Voelker KA, Pichot CS, Grange RW, Raphael RM, Klip A, Corey SJ: The F-BAR protein CIP4 promotes GLUT4 endocytosis through bidirectional interactions with N-WASp and Dynamin-2. J Cell Sci 2009, 122:2283-2291.

4. Feng Y, Hartig SM, Bechill JE, Blanchard EG, Caudell E, Corey SJ: The Cdc42 interacting protein-4 (CIP4) gene knock-out mouse reveals delayed and decreased endocytosis. J Biol Chem 2010, 285:4348-4354.

5. Tsujita K, Suetsugu S, Sasaki N, Furutani M, Oikawa T, Takenawa T: Coordination between the actin cytoskeleton and membrane deformation by a novel membrane tubulation domain of $\mathrm{PCH}$ proteins is involved in endocytosis. J Biol Chem 2006, 172:269-279.

6. Tian L, Nelson DL, Stewart DM: Cdc42-interacting protein 4 mediates binding of the Wiskott-Aldrich syndrome protein to microtubules. J Biol Chem 2000, 275:7854-7861.

7. Banerjee PP, Pandey R, Zheng R, Suhoski MM, Monaco-Shawver L, Orange JS: Cdc42-interacting protein-4 functionally links actin and microtubule networks at the cytolytic NK cell immunological synapse. $J$ Exp Med 2007, 204:2305-2320.

8. Yuan R, Fan S, Achary M, Stewart DM, Goldberg ID, Rosen EM: Altered gene expression pattern in cultured human breast cancer cells treated with hepatocyte growth factor/scatter factor in the setting of DNA damage. Cancer Res 2001, 61:8022-8031.

9. Holbert S, Dedeoglu A, Humbert S, Saudou F, Ferrante RJ, Neri C: Cdc42interacting protein 4 binds to huntingtin: neuropathologic and biological evidence for a role in Huntington's disease. Proc Natl Acad Sci USA 2003, 100:2712-2717

10. Hirata T, Cui YJ, Funakoshi T, Mizukami Y, Ishikawa Y, Shibasaki F, Matsumoto M, Sakabe T: The temporal profile of genomic responses and protein synthesis in ischemic tolerance of the rat brain induced by repeated hyperbaric oxygen. Brain Res 2007, 1130:214-222.

11. Roignot J, Taieb D, Suliman M, Dusetti NJ, lovanna JL, Soubeyran P: CIP4 is a new ArgBP2 interacting protein that modulates the ArgBP2 mediated control of WAVE1 phosphorylation and cancer cell migration. Cancer Lett 2010, 288:116-123.

12. Hu J, Troglio F, Mukhopadhyay A, Everingham S, Kwok E, Scita G, Craig AW: F-BAR-containing adaptor CIP4 localizes to early endosomes and regulates Epidermal Growth Factor Receptor trafficking and downregulation. Cell Signal 2009, 21:1686-1697.

13. Cahir-McFarland ED, Carter K, Rosenwald A, Giltnane JM, Henrickson SE, Staudt LM, Kieff E: Role of NF-kB in cell survival and transcription of latent membrane protein 1-expressing or Epstein-Barr virus latency IIIinfected cells. J Virol 2004, 78:4108-4119.

14. Jaenisch R, Bird A: Epigenetic regulation of gene expression: how the genome integrates intrinsic and environmental signals. Nat Genet 2003, 33:245-254.

15. Holliday R: Epigenetics: a historical overview. Epigenetics 2006, 1:76-80.

16. Turner BM: Defining an epigenetic code. Nat Cell Biol 2007, 9:2-6.

17. Guil S, Esteller M: DNA methylomes, histone codes and miRNAs: Tying it all together. Int J Biochem Cell Biol 2009, 41:87-95.

18. Leu YW, Yan PS, Fan M, Jin VX, Liu JC, Curran EM, Welshons W, Wei SH, Davuluri RV, Plass C, Nephew KP, Huang TH: Loss of estrogen receptor 
signaling triggers epigenetic silencing of downstream targets in breast cancer. Cancer Res 2004, 64:8184-8192.

19. Lee KD, Kuo TK, Whang-Peng J, Chung YF, Lin CT, Chou SH, Chen JR, Chen YP, Lee OK: In vitro hepatic differentiation of human mesenchymal stem cells. Hepatology 2004, 40:1275-1284.

20. Yan PS, Shi H, Rahmatpanah F, Hsiau TH, Hsiau AH, Leu YW, Liu JC, Huang TH: Differential distribution of DNA methylation within the RASSF1A CpG island in breast cancer. Cancer Res 2003, 63:6178-6186.

21. Jin VX, Leu YW, Liyanarachchi $S$, Sun $H$, Fan M, Nephew KP, Huang TH, Davuluri RV: Identifying estrogen receptor a target genes using integrated computational genomics and chromatin immunoprecipitation microarray. Nucleic Acids Res 2004, 32:6627-6635.

22. Hsiao SH, Lee KD, Hsu CC, Tseng MJ, Jin VX, Sun WS, Hung YC, Yeh KT, Yan PS, Lai YY, Sun HS, Lu YJ, Chang YS, Tsai SJ, Huang TH, Leu YW: DNA methylation of the Trip10 promoter accelerates mesenchymal stem cell lineage determination. Biochem Biophys Res Commun 2010, 400:305-12.

23. Colin E, Regulier E, Perrin V, Durr A, Brice A, Aebischer P, Deglon N, Humbert S, Saudou F: Akt is altered in an animal model of Huntington's disease and in patients. Eur J Neurosci 2005, 21:1478-1488.

24. Zhang Y, Rivera Rosado LA, Moon SY, Zhang B: Silencing of D4-GD inhibits growth and invasive behavior in MDA-MB-231 cells by activation of Rac-dependent p38 and JNK signaling. J Biol Chem 2009, 284:12956-12965.

25. The Cancer Genome Atlas Research Network: Comprehensive genomic characterization defines human glioblastoma genes and core pathways. Nature 2008, 455:1061-1068.

26. Liu P, Cheng H, Roberts TM, Zhao JJ: Targeting the phosphoinositide 3-kinase pathway in cancer. Nat Rev Drug Discov 2009, 8:627-644.

27. Gonzalez E, McGraw TE: The Akt kinases: isoform specificity in metabolism and cancer. Cell Cycle 2009, 8:2502-2508.

doi:10.1186/1423-0127-18-12

Cite this article as: Hsu et al:: Functional characterization of Trip10 in cancer cell growth and survival. Journal of Biomedical Science 2011 18:12.

\section{Submit your next manuscript to BioMed Central and take full advantage of:}

- Convenient online submission

- Thorough peer review

- No space constraints or color figure charges

- Immediate publication on acceptance

- Inclusion in PubMed, CAS, Scopus and Google Scholar

- Research which is freely available for redistribution

Submit your manuscript at www.biomedcentral.com/submit 